\title{
Utilização do método de Papadakis na melhoria da qualidade experimental de ensaios com soja
}

\author{
Lindolfo Storck ${ }^{(1)}$, Cleiton Steckling( ${ }^{(2)}$, Teresinha Roversi(2) e Sidinei José Lopes ${ }^{(1)}$
}

\begin{abstract}
(1)Universidade Federal de Santa Maria, Centro de Ciências Rurais, Departamento de Fitotecnia, CEP 97105-900 Santa Maria, RS. E-mail: lindolfo@smail.ufsm.br, sjlopes@smail.ufsm.br (2)Fundação Centro de Experimentação e Pesquisa, Rodovia RS 342, Km 149, CEP 98100-970 Cruz Alta, RS. E-mail: cleiton@fundacep.com.br, teresinha@fundacep.com.br
\end{abstract}

\begin{abstract}
Resumo - O trabalho teve como objetivo verificar as modificações na análise estatística, em relação ao atendimento aos pressupostos do modelo matemático e aos diferentes indicadores de precisão, que podem ser atribuídas à aplicação do método de Papadakis, na análise dos ensaios de competição de genótipos de soja. Foram usados os resultados da produção de grãos de soja de 226 ensaios de competição de genótipos, executados no delineamento de blocos completos ao acaso. Em cada ensaio, foram realizados: a verificação dos pressupostos, a análise da variância, os testes de hipóteses e outras estatísticas para identificação da precisão. Para verificar os ganhos em precisão, os mesmos dados foram analisados pelo método de Papadakis. Os pressupostos não foram violados em nenhum dos dois métodos de análise. A média do índice de diferenciação de Fasoulas aumentou de 5 para 12,43, em blocos homogêneos, e para 13,85, em blocos heterogêneos. O coeficiente de determinação, adequado para a classificação de precisão de experimentos, foi aproximadamente $10 \%$ superior com o uso do método de Papadakis, independentemente da eficiência do uso de blocos. A eficiência do método de Papadakis independe da eficiência do uso dos blocos e permite melhorias nos indicadores de precisão experimental.
\end{abstract}

Termos para indexação: Glycine max, pressupostos, precisão experimental, análise espacial.

\section{Use of the Papadakis method to improve soybean trial quality}

\begin{abstract}
The objective of this work was to verify the modifications in statistical analysis regarding the assumption fulfillment and the different precision indicators which can be attributed to the application of the Papadakis method, in the analysis of soybean grain yield experiments. The results of soybean yield of 226 trials of genotype competition, performed in a design of complete randomized blocks, were used. For each experiment, the assumptions were checked for: the variance analyses, hypothesis tests and other analysis to identify its precision. In order to verify the profit in precision, the same data were analyzed using the Papadakis method. The assumptions were adequate for both analysis types. The mean of Fasoulas differentiation index increased from 5 to 12.43, for homogeneous blocks, and to 13.85 , for heterogeneous blocks. The determination coefficient, adequate to classify the precision of the experiments, was approximately $10 \%$ higher, when the Papadakis method was used, regardless the efficiency of block use. The efficiency of the Papadakis method does not depend on the efficiency of the blocks, and allows an improvement of the indicators of experimental precision.
\end{abstract}

Index terms: Glycine max, assumptions, experimental precision, spatial analysis.

\section{Introdução}

Os pequenos aumentos de produtividade de grãos de soja (Glycine max L.), obtidos nos programas de melhoramento genético, são importantes porque são permanentes e dispensam novos investimentos. No entanto, muitas vezes, em razão da falta de precisão na avaliação dos genótipos sob seleção, esses pequenos aumentos não são identificados como significativos pelos métodos de análise estatística, o que faz com que genótipos promissores sejam erroneamente descartados do programa. Algumas técnicas experimentais, usadas no planejamento, condução e análise dos dados, podem melhorar a precisão dos resultados e evitar a perda de material genético com maior potencial de produtividade.

Para a melhoria da precisão experimental, o tamanho de parcela, o número de repetições, a escolha do delineamento e o uso de covariância são amplamente estudados. As fontes de erro experimental (heterogeneidade entre as observações nas unidades experimentais do mesmo tratamento) e o 
uso de técnicas experimentais adequadas para sua redução são descritos por Gomez \& Gomez (1984), Banzatto \& Kronka (1996), Steel et al. (1997), Ramalho et al. (2005) e Storck et al. (2006b). Vários autores realizaram estudos com aplicação de diferentes métodos para a determinação dessas técnicas, nas diferentes culturas agrícolas (Rossetti, 2002; Viana et al., 2002; Henriques Neto et al., 2004; Martin et al., 2005; Lopes et al., 2005; Oliveira et al., 2005, 2006; Storck et al., 2006a).

O uso, como covariável, dos valores das análises do solo (Feijó et al., 2001) ou da densidade de plantas (Cargnelutti Filho et al., 2006), de cada unidade experimental, foi estudado e proporcionou ganhos de precisão na cultura de milho. Alternativamente, diferentes métodos de análise espacial têm sido aplicados para aumentar a precisão experimental (Saez \& Magaña, 1990; Vivaldi, 1990; Amaro et al., 1997; Souza et al., 2000; Souza et al., 2003; Costa et al., 2005). O método de Papadakis (Papadakis, 1937) tem proporcionado ganhos de precisão em ensaios com milho (Cargnelutti Filho et al., 2003), desde que se tenham facilidades computacionais. Trata-se de um método de ajuste espacial, que utiliza as médias dos erros experimentais calculadas entre as parcelas vizinhas como covariável, a fim de diminuir a variância do erro experimental. Ainda não é bem conhecida a magnitude da eficiência do método de Papadakis para as diferentes culturas e ambientes. No entanto, este método possui uma precisão no mínimo igual à de outros métodos de análise espacial (Vivaldi, 1990).

Papadakis (1984) propôs modificações na sua metodologia, detalhada em Amaro \& Cobo (1994), pela inclusão de um procedimento de suavização das trocas repentinas de produtividade entre duas parcelas vizinhas, para eliminação das parcelas anormais, e essa modificação ainda não teve sua eficiência comprovada. No entanto, Cargnelutti Filho et al. (2003) estudaram diferentes abrangências de parcelas vizinhas, na estimativa do erro médio (covariável), e concluíram que o uso dos erros da vizinhança mais próxima é adequado e corresponde ao método original de Papadakis (1937).

Muitas vezes, o pesquisador tem dificuldades em identificar as variações de fertilidade existentes no local do experimento, para posicionar eficientemente os blocos. Nesses casos, o uso do delineamento inteiramente casualizado tem menor precisão, e o uso do delineamento de blocos ao acaso retira de forma ineficiente graus de liberdade do erro, o que também reduz a precisão experimental. Se for considerada a possibilidade do método de Papadakis ser eficiente para a substituição de blocos, até nos casos em que não se tem variação identificada entre os blocos, poderia ser adotado indiscriminadamente o delineamento inteiramente casualizado, aplicando-se a análise pelo método de Papadakis.

O objetivo deste trabalho foi verificar as modificações na análise estatística, em relação ao atendimento aos pressupostos do modelo matemático e aos diferentes indicadores de precisão, que podem ser atribuídos à aplicação do método de Papadakis, na análise dos ensaios de competição de genótipos de soja.

\section{Material e Métodos}

Foram usados os resultados de produtividade de grãos de soja de 226 ensaios de competição de genótipos ( $\mathrm{kg} \mathrm{ha}^{-1}$, ajustado a $13 \%$ de umidade). Os ensaios foram executados durante os anos agrícolas 2003/2004, 2004/ 2005 e 2005/2006, no conjunto de áreas experimentais (diferentes locais) coordenadas pela Fundação Centro de Experimentação e Pesquisa (Fundacep - Cruz Alta, Cachoeira do Sul e Restinga Seca) e pela Fundação Estadual de Pesquisa Agropecuária (Fepagro Sementes - Júlio de Castilhos, Santo Augusto, São Borja e Veranópolis), no Rio Grande do Sul. Foi usado o delineamento de blocos completos ao acaso, com número de repetições entre três e quatro, e com 10 a 20 genótipos. As unidades experimentais foram compostas por quatro linhas de $6 \mathrm{~m}$ de comprimento e $0,4 \mathrm{~m}$ entre linhas $\left(4 \mathrm{~m}^{2}\right.$ de área útil). Os ensaios foram classificados quanto ao estágio do melhoramento (preliminares, valor de cultivo e uso - VCU - e recomendadas) e quanto ao ciclo da cultura (precoce, médio e tardio). Os ensaios preliminares foram constituídos por genótipos de diferentes ciclos ou mistura de ciclos.

Em cada ensaio, procedeu-se: à análise de variância, com testes de hipóteses para blocos e genótipos, à estimativa da eficiência relativa do uso de blocos (em relação ao delineamento inteiramente casualizado), à estimativa da média por genótipo e média geral $(\hat{\mathrm{m}})$ e à amplitude $(\mathrm{H})$ entre as médias de genótipos (diferença entre a maior e menor média). Também, foram estimados, em cada ensaio, o coeficiente de variação (CV) e a diferença mínima significativa entre os genótipos pelo teste de Tukey, a 5\% de probabilidade (D), 
$\mathrm{D}=\mathrm{q}_{\left(\alpha ; \mathrm{I} ; \mathrm{GL}_{\mathrm{E}}\right)} \sqrt{\mathrm{QM}_{\mathrm{E}} / \mathrm{J}}$, em que $\mathrm{q}_{\left(\alpha ; \mathrm{I} ; \mathrm{GL}_{\mathrm{E}}\right)}$ é o valor da tabela para o teste de Tukey; I é o número de genótipos; $\mathrm{GL}_{\mathrm{E}}$ são os graus de liberdade do erro; $\mathrm{QM}_{\mathrm{E}}$ é a estimativa do erro experimental; e J expressa as repetições. A diferença mínima significativa entre os genótipos, pelo teste de Tukey, expresso em percentagem da média (DMS), foi obtida por $\mathrm{DMS}=100 \mathrm{D} / \hat{\mathrm{m}}$. Outras estatísticas adotadas na avaliação da precisão experimental (Storck et al., 2000) foram: o número de diferenças significativas, dentro da amplitude entre as médias (HD = H/D); e o índice de diferenciação de Fasoulas (IDF) (Fasoulas, 1983), estimado pela expressão:

$\mathrm{IDF}=200 \sum_{\mathrm{i}=1}^{\mathrm{n}} \mathrm{m}_{\mathrm{i}} /[\mathrm{n}(\mathrm{n}-1)]$,

em que $\mathrm{m}_{\mathrm{i}}$ é o número de médias que o i-ésimo genótipo supera estatisticamente, após a aplicação do teste de Tukey. Esse índice fornece o percentual de diferenças estatísticas, entre as médias, que o método de comparação múltipla de médias (Tukey) consegue detectar. Determinou-se o valor da estatística $\mathrm{r}^{2}$, designada por coeficiente de determinação (Cargnelutti Filho \& Storck, 2007), expressa por $\mathrm{r}^{2}=\mathrm{QM}_{\mathrm{g}} /\left(\mathrm{QM}_{\mathrm{g}}+\mathrm{QM}_{\mathrm{E}}\right)$, em que $\mathrm{QM}_{\mathrm{g}}$ é o quadrado médio de genótipos.

Foram estimados os valores do erro de cada unidade experimental, tendo-se considerado o delineamento de blocos ao acaso (valor observado menos a média geral, menos o efeito do bloco e menos o efeito do genótipo). Os valores do erro foram usados para os testes dos pressupostos da normalidade, aleatoriedade, homogeneidade das variâncias e aditividade (Marques et al., 2000). O teste de Lilliefors (Sprent \& Smeeton, 2007) foi aplicado, para se verificar a normalidade da distribuição dos erros. Para o teste da aleatoriedade dos erros, ou teste de seqüência (Sprent \& Smeeton, 2007), os erros, classificados em positivos e negativos, foram identificados pela seqüencia, de acordo com a casualização de cada ensaio, tendo-se começado pelo bloco 1, com numeração das unidades experimentais da esquerda para a direita, seguindo a numeração das unidades experimentais do bloco 2, da direita para a esquerda, e assim por diante, até o último bloco e a última unidade experimental. A estatística do teste é igual ao número de vezes em que é trocado um sinal por outro, ao percorrer a seqüência de erros estabelecidos, e foi testada pela aproximação da distribuição normal padrão.

O teste de Bartlett (Steel et al., 1997) foi aplicado para se verificar a homogeneidade das variâncias dos erros entre os genótipos. A aditividade do modelo matemático foi verificada pelo teste de não-aditividade, do modelo matemático de Tukey (Snedecor \& Cochran, 1989), a 5\% de probabilidade de erro.

Foram estimados, ainda, os erros, de acordo com o delineamento inteiramente casualizado (valor observado menos a média geral e menos o efeito de genótipo). Com esses valores, foi estimado o erro médio de cada unidade experimental como sendo a média entre o erro da unidade considerada e os erros das unidades experimentais vizinhas (lado direito, lado esquerdo, da frente e do fundo) existentes. $\mathrm{O}$ valor do erro médio foi denominado de covariável, para fins da análise da covariância, segundo o delineamento inteiramente casualizado (Steel et al., 1997; Cargnelutti Filho et al., 2003), o que corresponde à aplicação do método de Papadakis (1937).

Os valores da covariável foram submetidos à análise de variância, segundo o modelo do delineamento de blocos ao acaso, e testados quanto aos pressupostos da normalidade, aleatoriedade e homogeneidade das variâncias. Foi estimada a amplitude, entre os valores da covariável, e a amplitude entre as médias de genótipos para a covariável.

A análise de covariância, com testes de hipóteses dos efeitos da covariável e de genótipos, foi feita conforme Steel et al. (1997), e repetiu as estimativas de amplitude (H) entre médias de genótipos ajustadas, CV, D, DMS, HD, IDF e $r^{2}$. Também foi estimada a eficiência relativa do uso da covariável, em relação ao uso do delineamento de blocos ao acaso, e foram testadas as hipóteses dos pressupostos (normalidade, aleatoriedade e homogeneidade das variâncias), referentes ao erro neste modelo, e do paralelismo entre os genótipos, quanto à resposta da covariável (Seber, 1976; Storck et al., 2000). Por fim, foi testada a hipótese de que o método de Papadakis reduz a variância do erro experimental, em relação ao delineamento de blocos ao acaso, por meio da estatística $\mathrm{F}=\mathrm{QM}_{\mathrm{E}} / \mathrm{QM}_{\mathrm{EA}}$, em que: $\mathrm{QM}_{\mathrm{E}}$ é o quadrado médio do erro da análise de variância com delineamento de blocos ao acaso; e $\mathrm{QM}_{\mathrm{EA}}$ é o quadrado médio do erro, ajustado pelo método de Papadakis.

Para os cálculos, foi elaborado e compilado um programa específico com linguagem Pascal. 


\section{Resultados e Discussão}

A percentagem de ensaios com blocos significativamente heterogêneos, a $5 \%$ de probabilidade de erro, foi baixa $(28,8 \%)$, seja porque as áreas experimentais eram homogêneas, seja porque os blocos não foram adequadamente posicionados. Mesmo assim, os genótipos foram significativamente diferentes em $76,1 \%$ dos ensaios (Tabela 1). Os pressupostos (normalidade, aleatoriedade, homogeneidade das variâncias e aditividade) do modelo matemático, para o delineamento experimental adotado (blocos ao acaso), não foram violados para a maioria dos ensaios e não se constituíram em motivo de restrições para as interpretações. Marques et al. (2000), em 307 ensaios com milho, verificaram violação dos pressupostos da normalidade, homogeneidade e aditividade equivalentes e uma proporção maior de violação da aleatoriedade.

Apenas em 0,9\% dos ensaios, a covariável, média do erro experimental na vizinhança, foi significativamente dependente do efeito de genótipos (Tabela 1). Este fato mostra que as diferenças entre os tratamentos independem dos valores da covariável, pré-requesito para se proceder à análise da covariância e ajustar as médias de genótipos em função das médias da covariável (Steel et al., 1997). Também, os pressupostos da aleatoriedade e homogeneidade das variâncias foram adequados em todos os casos. No entanto, a falta de normalidade foi observada em 16,4\% dos casos, aproximadamente o dobro dos casos observados $(8,4 \%)$ para o erro da variável dependente (produção de grãos).

A média de produtividade de grãos, obtida nos 226 ensaios, variou entre $298 \mathrm{~kg} \mathrm{ha}^{-1} \mathrm{e} 3.489 \mathrm{~kg} \mathrm{ha}^{-1}$, com

Tabela 1. Percentagens de casos em que diferentes hipóteses da nulidade foram rejeitadas para a análise de variância (ANOVA), para a covariável do método de Papadakis e para a análise pelo método de Papadakis, em 226 ensaios de competição de genótipos de soja.

\begin{tabular}{lccc}
\hline Hipótese sobre & ANOVA & Covariável & Papadakis \\
\hline Blocos & 28,8 & 38,9 & - \\
Genótipos & 76,1 & 0,9 & 96,9 \\
Normalidade (N) & 8,4 & 16,4 & 8,4 \\
Aleatoriedade (Al) & 0,9 & 0,0 & 0,0 \\
Homogeneidade (H) & 0,0 & 0,0 & 0,4 \\
Aditividade (Ad) & 9,7 & - & - \\
N e Al & 0,0 & 0,0 & 0,0 \\
N e H & 0,0 & 0,0 & 0,0 \\
N e Ad & 0,9 & - & - \\
Al e H & 0,0 & 0,0 & 0,0 \\
Al e Ad & 0,0 & - & - \\
H e Ad & 0,0 & - & - \\
\hline
\end{tabular}

média geral igual a $1.921 \mathrm{~kg} \mathrm{ha}^{-1} \mathrm{e}$ coeficiente de variação entre as médias dos ensaios igual a 33,65\% (Tabela 2). Esta variabilidade entre os ensaios é favorável para o estudo do efeito do uso do método de Papadakis, porque envolve uma ampla faixa de condições ambientais e diversidade de genótipos (preliminares, VCU e recomendadas) e de ciclos (Tabela 3). Nos ensaios preliminares, não houve agrupamento de genótipos de mesmo ciclo em cada ensaio, porque não foram avaliados para esta característica.

A média dos coeficientes de variação, observada nos ensaios (13,74\%), foi menos de $2 \%$ superior à relatada por Lúcio et al. (1999), em estudo com 480 ensaios de soja. No presente estudo, a média dos $\mathrm{CV}$ foi um pouco maior em razão, possivelmente, da grande participação de ensaios preliminares (68\%), com genótipos de diferentes ciclos (mistura de ciclos), o que não é o caso dos ensaios de genótipos recomendados ou os de VCU. A amplitude de variação dos CV $(47,2 \%)$ também é importante para o estudo, pois tem diversidade na precisão dos ensaios.

Para os 28,8\% dos ensaios em que os blocos foram significativamente heterogêneos, a média da eficiência relativa foi igual a $134,8 \%$, o que significa que, caso fosse adotado o delineamento inteiramente ao acaso, com o mesmo número de repetições, $28,8 \%$ dos ensaios possuiriam estimativa da variância da média de genótipo $34,8 \%$ maior. Em princípio, mesmo sendo baixo o número de ensaios com significância para blocos, não se poderia descartar um ganho em precisão, nestes casos, proporcionado pela sua adoção.

As amplitudes e o coeficiente de variação dos valores da covariável (Tabela 2), entre as unidades experimentais (Amplitude U) e entre as médias de genótipos (Amplitude M), foram adequadas para o estudo, visto que atenderam a uma ampla faixa de variação.

A análise dos dados pelo método de Papadakis aumentou o número de casos em que o efeito de genótipos foi significativo, de $76,1 \%$ para $96,9 \%$ (Tabela 1) e, em $100 \%$ dos casos, o efeito da covariável (efeito do coeficiente de regressão linear) foi significativo, a 5\% de probabilidade de erro. Portanto, mesmo nos $71,2 \%$ dos casos em que o efeito de blocos não foi significativo, a covariável foi significativa e, com isto, as médias dos genótipos podem ser ajustadas pela covariável, em razão da eficiência do método. Outro resultado favorável ao método de Papadakis é que os pressupostos (normalidade, aleatoriedade e homogeneidade das variâncias) do erro, com o modelo da análise de covariância, foram violados em proporções semelhantes ao da aplicação do modelo do delineamento de blocos ao acaso (Tabela 1). 
Os valores dos coeficientes de regressão linear (efeito da covariável) tiveram variação entre 0,8569 e 2,7637, para blocos homogêneos, e menor amplitude em condição de blocos heterogêneos (Tabela 2). Isto evidencia que os valores da produção de grãos puderam ser ajustados significativamente, em $100 \%$ dos ensaios, para mais ou para menos, com intensidades variadas nos diferentes ensaios.

Ao se compararem os indicadores de precisão experimental (CV, D, DMS, HD, IDF e r²), obtidos com o uso do método de Papadakis, nas condições de blocos homogêneos e heterogêneos, observou-se um incremento generalizado na qualidade dos indicadores de precisão, em relação aos mesmos indicadores da análise usual (blocos ao acaso). Assim, mesmo nos casos em que os blocos eram homogêneos, o método de Papadakis melhorou a precisão experimental dos ensaios. Deve ser considerado o fato de que, ao se aplicar o método de Papadakis, são somados dois ou três graus de liberdade aos do erro, em razão da retirada da fonte de variação de blocos da análise de variância (três ou quatro blocos, respectivamente) e da diminuição de um grau de liberdade, em razão do efeito linear da covariável. Este incremento nos GL do erro reduz a sua variância, nos casos de blocos homogêneos.

A eficiência relativa do uso do método de Papadakis, em relação ao uso do delineamento de blocos ao acaso, foi de $116,6 \%$ (blocos heterogêneos), com variação entre

Tabela 2. Valor mínimo, valor máximo, amplitude, média e coeficiente de variação (CV) de diferentes estatísticas estimadas em ensaios de competição de genótipos de soja (produtividade de grãos, $\mathrm{t}$ ha ${ }^{-1}$ ) e relacionadas à aplicação do método de Papadakis.

\begin{tabular}{|c|c|c|c|c|c|}
\hline Estatística $^{(1)}$ & Mínimo & Máximo & Amplitude & Média & $\mathrm{CV}(\%)$ \\
\hline & \multicolumn{5}{|c|}{ Sem o uso do método de Papadakis (226 ensaios) } \\
\hline ER (\%) & 112,3 & 215,0 & 102,7 & 134,8 & 18,93 \\
\hline Média $\left(\mathrm{t} \mathrm{ha}^{-1}\right)$ & 0,2980 & 3,4889 & 3,1909 & 1,9207 & 33,65 \\
\hline $\mathrm{CV}(\%)$ & 3,50 & 50,70 & 47,20 & 13,74 & 41,97 \\
\hline D Tukey & 0,2458 & 1,8413 & 1,5955 & 0,7430 & 37,14 \\
\hline Amplitude M & 0,2583 & 2,0542 & 1,7959 & 0,8655 & 36,29 \\
\hline DMS $(\%)$ & 10,5 & 153,6 & 143,1 & 41,5 & 42,58 \\
\hline HD & 0,62 & 2,56 & 1,94 & 1,23 & 33,57 \\
\hline IDF & 0,00 & 40,52 & 40,52 & 5,00 & 151,66 \\
\hline \multirow[t]{2}{*}{$\mathrm{r}^{2}(\%)$} & 50,6 & 93,7 & 43,1 & 72,8 & 14,42 \\
\hline & \multicolumn{5}{|c|}{ Covariável do método de Papadakis } \\
\hline Amplitude U & 0,1812 & 1,8031 & 1,6219 & 0,5182 & 42,02 \\
\hline \multirow[t]{2}{*}{ Amplitude M } & 0,0342 & 0,6511 & 0,6169 & 0,1907 & 48,22 \\
\hline & \multicolumn{5}{|c|}{ Com o uso do método de Papadakis - blocos homogêneos (161 ensaios) } \\
\hline Beta & 0,8569 & 2,7637 & 1,9068 & 1,4391 & 16,16 \\
\hline CV (\%) & 3,7 & 27,5 & 23,8 & 10,1 & 37,70 \\
\hline D Tukey & 0,1551 & 1,2033 & 1,0482 & 0,5617 & 37,51 \\
\hline Amplitude M & 0,2680 & 1,7714 & 1,5034 & 0,8872 & 36,02 \\
\hline DMS (\%) & 11,6 & 85,3 & 73,7 & 30,7 & 37,83 \\
\hline $\mathrm{HD}$ & 0,80 & 3,76 & 2,96 & 1,68 & 33,40 \\
\hline IDF & 0,00 & 45,10 & 45,10 & 12,43 & 88,48 \\
\hline$r^{2}(\%)$ & 60,4 & 96,2 & 35,8 & 82,5 & 9,44 \\
\hline \multirow[t]{2}{*}{ ER P (\%) } & 53,2 & 203,8 & 150,6 & 98,1 & 31,14 \\
\hline & \multicolumn{5}{|c|}{ Com o uso do método de Papadakis - blocos heterogêneos (65 ensaios) } \\
\hline Beta & 0,9292 & 2,0638 & 1,1346 & 1,4423 & 14,01 \\
\hline $\mathrm{CV}(\%)$ & 2,4 & 38,6 & 36,2 & 10,1 & 46,34 \\
\hline D Tukey & 0,2142 & 1,4088 & 1,1946 & 0,5383 & 42,61 \\
\hline Amplitude M & 0,3037 & 2,0668 & 1,7631 & 0,8208 & 35,72 \\
\hline DMS (\%) & 7,4 & 117,5 & 110,1 & 30,5 & 46,83 \\
\hline HD & 0,75 & 3,60 & 2,85 & 1,64 & 34,34 \\
\hline IDF & 0,00 & 52,38 & 52,38 & 13,85 & 96,35 \\
\hline$r^{2}(\%)$ & 60,4 & 96,9 & 36,5 & 82,1 & 10,56 \\
\hline ER P $(\%)$ & 59,8 & 208,6 & 148,8 & 116,6 & 28,88 \\
\hline
\end{tabular}

${ }^{(1)}$ ER, eficiência relativa do uso de blocos; CV, coeficiente de variação do erro experimental; D Tukey, diferença mínima significativa pelo teste de Tukey, a 5\% de probabilidade; amplitude entre médias (M) e entre dados (U); DMS, D Tukey em percentagem da média; HD, amplitude/D Tukey; IDF, índice de diferenciação de Fasoulas; $\mathrm{r}^{2}$, coeficiente de determinação do modelo; Beta, efeito da covariável, coeficiente de regressão linear; ER P, eficiência relativa do uso do método de Papadakis. 
Tabela 3. Número de ensaios (n), média da eficiência relativa (\%) do uso do método de Papadakis, e número de ensaios com respostas não paralelas para a covariável (nP), na análise de ensaios de competição de genótipos de soja, na condição de blocos heterogêneos e homogêneos, classificados quanto ao ciclo (precoce, médio, tardio e mistura) e quanto à significância do método de Papadakis (Pap Ho e Pap H1).

\begin{tabular}{|c|c|c|c|c|c|c|}
\hline \multirow[t]{2}{*}{ Ciclo } & \multicolumn{3}{|c|}{ Blocos heterogêneos } & \multicolumn{3}{|c|}{ Blocos homogêneos } \\
\hline & $\mathrm{n}$ & Média & $\mathrm{nP}$ & $\mathrm{n}$ & Média & $\mathrm{nP}$ \\
\hline Precoce & 11 & 125,8 & 5 & 14 & 89,6 & 11 \\
\hline Médio & 11 & 112,8 & 8 & 14 & 115,2 & 8 \\
\hline Tardio & 4 & 105,5 & 3 & 18 & 84,8 & 10 \\
\hline Mistura & 39 & 116,2 & 18 & 115 & 99,1 & 48 \\
\hline Pap Ho $(45 \%)$ & 35 & 96,8 & - & 66 & 73,5 & - \\
\hline Pap H1 (55\%) & 30 & 139,7 & - & 95 & 115,1 & - \\
\hline Geral & 65 & 116,6 & 34 & 161 & 98,1 & 77 \\
\hline
\end{tabular}

os ciclos (Tabela 3) - maior para o ciclo precoce e menor para o ciclo tardio. De forma semelhante, Souza et al. (2000), ao analisar ensaios com feijão, obtiveram eficiência semelhante à proporcionada pela análise em látice do método de Papadakis, quanto ao controle da heterogeneidade dos blocos.

O método de Papadakis reduziu significativamente a variância do erro experimental, o que fez com que a variância do erro ajustada fosse significativamente menor, se comparada ao uso do delineamento de blocos ao acaso, em $55 \%$ dos ensaios, e fosse mantida, aproximadamente, a proporção entre blocos homogêneos e heterogêneos. Esta constatação é favorável ao uso do método de Papadakis, pois em apenas $28,8 \%$ dos ensaios os blocos foram eficientes.

Pelos resultados obtidos, a eficiência do método de Papadakis independe da eficiência do uso dos blocos. O que realmente é importante a ser observado são as melhorias nos indicadores de precisão experimental. Por exemplo, a média do índice de diferenciação de Fasoulas (IDF, Tabela 2) aumentou de 5 para 12,43 nos blocos homogêneos, e para 13,85 nos blocos heterogêneos, e discriminou os genótipos em maior número de grupos, o que facilita a decisão dos melhoristas. Também, o coeficiente de determinação $\left(\mathrm{r}^{2}\right)$, adequado para a classificação de precisão de experimentos (Cargnelutti Filho \& Storck, 2007), foi aproximadamente $10 \%$ superior com o uso do método de Papadakis, independentemente da eficiência do uso de blocos.

Os experimentadores têm dificuldades em posicionar os blocos adequadamente ou, então, os usam de forma indiscriminada. Neste caso, como a eficiência do método de Papadakis independe da eficiência do uso de blocos, seria recomendável o uso do delineamento inteiramente ao acaso, com a análise estatística realizada pelo método de Papadakis, sem que isso acarretasse em prejuízos aos pressupostos, além dos ganhos na precisão experimental e na qualidade da discriminação do material genético sob avaliação.

No entanto, causam preocupação os $49,1 \%$ dos ensaios (34 ensaios com blocos heterogêneos e 77 com blocos homogêneos) em que os coeficientes de regressão não são paralelos entre os diferentes genótipos (Tabela 3). A falta de paralelismo indica que os genótipos de um mesmo ensaio têm suas médias ajustadas em intensidades variadas, a depender do coeficiente de regressão de cada genótipo. Genótipos com maior sensibilidade à variação ambiental têm maior coeficiente de regressão e maior influência no ajuste da média desses genótipos. No entanto, o ajuste das médias é realizado pelo mesmo coeficiente de regressão, para todos os genótipos. Assim, se poderia pensar em ajustar as médias pela covariável, de acordo com algum tipo de estratificação de genótipos.

\section{Conclusões}

1. A análise da produtividade de grãos, dos ensaios de competição de genótipos de soja, pelo método de Papadakis, mantém adequados os pressupostos de normalidade, aleatoriedade, homogeneidade e aditividade, quando comparada com o modelo do delineamento de blocos ao acaso, sejam os blocos homogêneos ou heterogêneos.

2. O método de Papadakis, comparado ao uso do modelo blocos ao acaso, melhora as estatísticas que retratam a precisão experimental.

\section{Referências}

AMARO, R.; COBO, M. La investigación agrícola y la aplicación del método de Papadakis. Revista de la Faculdad de Agronomía, v.20, p.35-45, 1994. 
AMARO, R.; COBO, M.; QUIROZ, A. Comparación de métodos de ajuste en el análisis espacial de experimentos de campo. Agronomía Tropical, v.47, p.359-374, 1997.

BANZATTO, D.A.; KRONKA, S.N. Experimentação agrícola. 2.ed. Jaboticabal: Funep, 1996. 247p.

CARGNELUTTI FILHO, A.; STORCK, L. Estatísticas de avaliação da precisão experimental em ensaios de cultivares de milho. Pesquisa Agropecuária Brasileira, v.42, p.17-24, 2007.

CARGNELUTTI FILHO, A.; STORCK, L.; LOPES, S.J.; LÚCIO, A.D. Interferência da variabilidade da população de plantas de milho sobre a precisão experimental. Ciência Rural, v.36, p.42-50, 2006. CARGNELUTTI FILHO, A.; STORCK, L.; LÚCIO, A.D. Ajustes de quadrado médio do erro em ensaios de competição de cultivares de milho pelo método de Papadakis. Pesquisa Agropecuária Brasileira, v.38, p.467-473, 2003.

COSTA, J.R.; BUENO FILHO, J.S.S.; RAMALHO, M.A.P. Análise espacial e de vizinhança no melhoramento genético de plantas. Pesquisa Agropecuária Brasileira, v.40, p.1073-1079, 2005.

FASOULAS, A.C. Rating cultivars and trials in applied plant breeding. Euphytica, v.32, p.939-943, 1983.

FEIJÓ, S.; STORCK, L.; LÚCIO, A.D.; LOVATO, C.; MARTIN, T.N. Melhoria da qualidade de ensaios de avaliação de cultivares de milho através de indicadores de fertilidade do solo. Ciência Rural, v.31, p.225-230, 2001.

GOMEZ, K.A.; GOMEZ, A.A. Statistical procedures for agricultural research. 2.ed. New York: John Wiley, 1984. 680p.

HENRIQUES NETO, D.; SEDIYAMA, T.; SOUZA, M.A.; CECON, P.R.; YAMANAKA, C.H.; SEDIYAMA, M.A.N.; VIANA, A.E.S. Tamanho de parcelas em experimentos com trigo irrigado sob plantio direto e convencional. Pesquisa Agropecuária Brasileira, v.39, p.517-524, 2004.

LOPES, S.J.; STORCK, L.; LÚCIO, A.D.; LORENTZ, L.H.; LOVATO, C.; DIAS, V.O. Tamanho de parcela para produtividade de grãos de sorgo granífero em diferentes densidades de plantas. Pesquisa Agropecuária Brasileira, v.40, p.525-530, 2005.

LÚCIO, A.D.; STORCK, L.; BANZATTO, D.A. Classificação dos experimentos de competição de cultivares quanto a sua precisão. Pesquisa Agropecuária Gaúcha, v.5, p.99-103, 1999.

MARQUES, D.G.; STORCK, L.; LOPES, S.J.; MARTIN, T.N. Qualidade dos ensaios de competição de cultivares de milho no Estado do Rio Grande do Sul. Ciência Rural, v.30, p.381-385, 2000.

MARTIN, T.N.; DUTRA, L.M.C.; JAUER, A.; STORCK, L.; ZABOT, L.; UHRY, D.; SANTI, A.L.; STEFANELO, C.; LUCCA FILHO, O.A. Tamanho ótimo de parcela e número de repetições em soja (Glycine max L.). Ciência Rural, v.35, p.271-276, 2005. OLIVEIRA, S.J.R.; STORCK, L.; LOPES, S.J.; LÚCIO, A.D.; FEIJÓ, S.; DAMO, H.P. Plot size and experimental unit relationship in exploratory experiments. Scientia Agricola, v.62, p.585-589, 2005.
OLIVEIRA, S.J.R.; STORCK, L.; LÚCIO, A.D.; LOPES, S.J.; MARTINI, L.F.D. Índice de heterogeneidade, coeficiente de variação e tamanho ótimo de parcela em batata. Ciência Rural, v.36, p.1710-1716, 2006.

PAPADAKIS, J.S. Advances in the analysis of field experiments. Proceedings of the Academy of Athens, v.59, p.326-342, 1984.

PAPADAKIS, J.S. Méthod statistique pour des experiences sur champ. Thessalonike: Institut d'Amélioration des Plantes à Salonique, 1937. 30p. (Bullettin, 23).

RAMALHO, M.A.P.; FERREIRA, D.F.; OLIVEIRA, A.C. Experimentação em genética e melhoramento de plantas. 2.ed. Lavras: Ufla, 2005. 322p.

ROSSETTI, A.G. Influência da área da parcela e do número de repetições na precisão de experimentos com arbóreas. Pesquisa Agropecuária Brasileira, v.37, p.433-438, 2002.

SAEZ, T.C.; MAGAÑA, J.A.J. El análisis de experimentos por método Papadakis. Chapingo, v.15, p.110-113, 1990.

SEBER, G.A.F. Linear regression analysis. New York: John Wiley \& Sons, 1976. 465p.

SNEDECOR, G.W.; COCHRAN, W.G. Statistical methods. 8.ed. Ames: Iowa State University Press, 1989. 503p.

SOUZA, E.A.; GERALDI, I.O.; RAMALHO, M.A.P. Alternativas experimentais na avaliação de famílias em programas de melhoramento genético do feijoeiro. Pesquisa Agropecuária Brasileira, v.35, p.1765-1771, 2000.

SOUZA, E.A.; GERALDI, I.O.; RAMALHO, M.A.P.; BERTOLUCCI, F.L.G. Experimental alternatives for evaluation of progenies and clones in eucalyptus breeding programs. Revista Árvore, v.27, p.427-434, 2003.

SPRENT, P.; SMEETON, N.C. Applied nonparametric statistical methods. $4^{\text {th }}$ ed. Boca Raton: Chapman \& Hall, 2007. 530p.

STEEL, R.G.D.; TORRIE, J.H.; DICKEY, D.A. Principles and procedures of statistics: a biometrical approach. $3^{\text {rd }}$ ed. New York: McGraw Hill Book, 1997. 666p.

STORCK, L.; BISOGNIN, D.A.; OLIVEIRA, S.J.R. Dimensões dos ensaios e estimativas do tamanho ótimo de parcela em batata. Pesquisa Agropecuária Brasileira, v.41, p.903-909, 2006a.

STORCK, L.; GARCIA, D.C.; LOPES, S.J.; ESTEFANEL, V. Experimentação vegetal. 2.ed. Santa Maria: UFSM, 2006b. 198p. STORCK, L.; LOPES, S.J.; MARQUES, D.G.; TISOTT, C.A.; ROS, C.A. Análise de covariância para melhoria da capacidade de discriminação em ensaios de cultivares de milho. Pesquisa Agropecuária Brasileira, v.35, p.1311-1316, 2000.

VIANA, A.E.S.; SEDIYAMA, T.; CECON, P.R.; LOPES, S.C.; SEDIYAMA, M.A.N. Estimativas de tamanho de parcela em experimentos com mandioca. Horticultura Brasileira, v.20, p.58-63, 2002.

VIVALDI, L.J. Comparação entre métodos de análise espacial de experimentos de campo. Pesquisa Agropecuária Brasileira, v.25, p.77-84, 1990.

Recebido em 21 de fevereiro de 2008 e aprovado em 12 de maio de 2008 